\title{
STURMIAN THEORY AND DISCONJUGACY OF SECOND ORDER SYSTEMS
}

\begin{abstract}
FOZI M. DANNAN ${ }^{1}$
Abstract. A generalization of the Sturm Comparison Theorem is given to second order linear systems. In addition, an analogue to Sternberg disconjugacy criterion for nonselfadjoint second order linear systems is given.
\end{abstract}

1. Introduction. Consider the vector differential equations

$$
y^{\prime \prime}+A(t)=0
$$

and

$$
x^{\prime \prime}+B(t) x=0,
$$

where $A(t)=\left(a_{i j}(t)\right)$ and $B(t)=\left(b_{i j}(t)\right)$ are continuous $n \times n$ matrices. A number $\beta$ is a conjugate point of $\alpha, \alpha<\beta$, relative to (1) if (1) has a nontrivial solution vanishing at $\alpha$ and $\beta$. If $\beta$ is a conjugate point of $\alpha$ and if there is no number $\gamma$, $\alpha<\gamma<\beta$, such that $\gamma$ is also a conjugate point of $\alpha$, then $\beta$ is the first conjugate of $\alpha$. If no nontrivial solution of (1) vanishes twice in an interval $I$, then (1) is disconjugate on $I$.

Recently Ahmad and Lazer [2] gave a generalization of the Sturm Comparison Theorem [5] for nonselfadjoint systems. They showed that if $a_{i j}(t) \geqslant b_{i j}(t) \geqslant 0$, $1 \leqslant i, j \leqslant n$, with $a_{i j}(\bar{t})>b_{i j}(\bar{t})$ at some point $\bar{t}, \bar{t} \in[\alpha, \beta]$ (where $\beta$ is a conjugate point of $\alpha$ relative to (2)), then there exists a conjugate point $\gamma, \alpha<\gamma<\beta$, of $\alpha$ relative to (1). Later, in [3] the condition $a_{i j}(\bar{t})>b_{i j}(\bar{t}), 1 \leqslant i, j \leqslant n$, is relaxed to $a_{i i}(\bar{t})>b_{i i}(\bar{t}), 1 \leqslant i \leqslant n$, with the additional assumption that $A(t)$ and $B(t)$ be symmetric. The purpose of our first theorem is to show that this result holds when we replace the condition $a_{i j}(t) \geqslant b_{i j}(t) \geqslant 0,1 \leqslant i, j \leqslant n$, by the conditions $a_{i j}(t) \geqslant$ $b_{i j}(t)$ and $b_{i j}(t)+b_{j i}(t) \geqslant 0$ with the restriction that only $A(t)$ be symmetric.

In [4] it was shown by Sternberg that (1) is disconjugate on an interval $I$ iff there exists an $n \times n$ symmetric differentiable matrix $V(t)$ such that $V^{\prime}(t)+V^{2}(t)+A(t)$ is a nonpositive definite matrix, where $A(t)$ is symmetric. This result is the matrix analogue for the work of Wintner [6] for the scalar second order differential equations. Our second purpose of this note is to prove a similar theorem, where we assume that the elements of $V^{\prime}(t)+V^{2}(t)+A(t)$ are nonpositive. Our theorem neither implies the theorem of Sternberg nor is it implied by it.

Received by the editors December 20, 1982.

1980 Mathematics Subject Classification. Primary 34A25, Secondary 34C10.

Key words and phrases. Conjugate point, disconjugate, symmetric, positive matrix.

'Part of this work was done while the author was visiting the University of Miami, Coral Gables, Florida. 


\section{Main results.}

THEOREM 1. Let $A(t)$ and $B(t)$ be continuous $n \times n$ matrices defined on $[a, b]$, and $A(t)$ symmetric. Assume that

$$
a_{i j}(t) \underset{z}{z} b_{i j}(t)
$$

for $1 \leqslant i, j \leqslant n, a_{i i}(\bar{t})>b_{i i}(\bar{t})$ for some $\bar{t} \in(a, b), 1 \leqslant i \leqslant n$, and

$$
b_{i j}(t)+b_{j i}(t) \geqslant 0
$$

for $i \neq j$ and $t \in[a, b]$. If there exists a nontrivial solution of (2) such that $x(a)=$ $x(b)=0$, then there exists a nontrivial solution of (1) such that $y(a)=y(c)=0$, where $c$ is some number in $(a, b)$.

Proof. Since $x(t)$ is a solution of (2) with $x(a)=x(b)=0$, then

$$
\int_{a}^{b}\left(\left\langle x^{\prime}, x^{\prime}\right\rangle-\langle B x, x\rangle\right) d t=0
$$

Let $J[A, h ; a, b]$ define the functional

$$
J[A, h ; a, b]=\int_{a}^{b}\left(\left\langle h^{\prime}, h^{\prime}\right\rangle-\langle A h, h\rangle\right) d t
$$

where $h(t)$ belongs to the class $\mathbb{Q}[a, b]$ of absolutely continuous $R^{n}$-valued functions on $[a, b]$ such that $\left|h^{\prime}\right| \in L[a, b]$ and $h(a)=h(b)=0$. If we put

$$
u(t)=\left(\left|x_{1}(t)\right|, \ldots,\left|x_{n}(t)\right|\right),
$$

then $u(t) \in \mathbb{Q}[a, b]$. Let $\tilde{B}(t)=\frac{1}{2}\left[B(t)+B^{T}(t)\right]$. Then

$$
\begin{aligned}
J[A, u ; a, b] & =\int_{a}^{b}\left(\left\langle u^{\prime}, u^{\prime}\right\rangle-\langle A u, u\rangle\right) d t<\int_{a}^{b}\left(\left\langle u^{\prime}, u^{\prime}\right\rangle-\langle\tilde{B} u, u\rangle\right) d t \\
& \leqslant \int_{a}^{b}\left(\left\langle x^{\prime}, x^{\prime}\right\rangle-\langle\tilde{B} x, x\rangle\right) d t=\int_{a}^{b}\left(\left\langle x^{\prime}, x^{\prime}\right\rangle-\langle B x, x\rangle\right) d t=0 .
\end{aligned}
$$

Hence by Lemma 2 of [1] there exists a nontrivial solution for (1) which vanishes at $a$ and at a point $c \in(a, b)$.

The following example shows that the preceding theorem is false when $A(t)$ is not symmetric.

EXAMPLE 1. Let

$$
A=\left(\begin{array}{cc}
2 & -2 \\
4 & 2
\end{array}\right) \quad \text { and } B=\left(\begin{array}{cc}
2 & -2 \\
\frac{5}{2} & -4
\end{array}\right) .
$$

The equation $x^{\prime \prime}+B x=0$ has the solution $x(t)=\sin t\left(\begin{array}{l}2 \\ 1\end{array}\right)$, which vanishes at $t=0$ and $t=\pi$. We show that for any $a$ and $b$ with $b>a$, there does not exist $u(t)$ satisfying $u^{\prime \prime}+A u=0, u(a)=u(b)=0, u(t) \neq 0$. Suppose that such a solution $u(t)$ exists. If we let $u(t)=\operatorname{col}\left(u_{1}(t), u_{2}(t)\right)$, then

$$
u_{1}^{\prime \prime}+2 u_{1}=2 u_{2}, \quad u_{2}^{\prime \prime}+2 u_{2}=-4 u_{1} \text {. }
$$

Hence

$$
\left(u_{1}^{\prime} u_{2}-u_{1} u_{2}^{\prime}\right)^{\prime}=2 u_{2}^{2}+4 u_{1}^{2}
$$


Integration from $a$ to $b$ on both sides gives

$$
0=\int_{a}^{b}\left(2 u_{2}^{2}+4 u_{1}^{2}\right) d t .
$$

Therefore, we must have $u_{1}=u_{2} \equiv 0$ in $[a, b]$.

THEOREM 2. Let $A(t)$ be an $n \times n$ continuous matrix defined on $I=[a, b]$, such that $a_{i j}(t) \geqslant 0$ for $1 \leqslant i, j \leqslant n$ and $t \in[a, b]$. Then (1) is disconjugate on I if there exists $a$ continuously differentiable matrix $M(t)$ such that

$$
l_{i j}(t) \leqslant 0
$$

for $1 \leqslant i, j \leqslant n$ and $t \in I$, where

$$
\left(l_{i j}(t)\right)=L(t)=M^{\prime}(t)+\tilde{M}^{2}(t)+A(t) .
$$

Proof. Assume that (1) is not disconjugate on $[a, b]$. Then there exists a nontrivial solution $z(t)$ of (1) such that $z\left(t_{1}\right)=z\left(t_{2}\right)=0, t_{1}, t_{2} \in[a, b]$. From Lyaponov inequality for systems, it follows that there exists a first conjugate point $t_{0}$ of $t_{1}$ relative to (1). From [2, Theorem 4] it follows that there exists a nontrivial solution $u(t)$ of (1) such that $u\left(t_{1}\right)=u\left(t_{0}\right)=0$ and $u_{k}(t) \geqslant 0, k=1, \ldots, n$ and $t \in\left[t_{1}, t_{0}\right]$. If $M(t)$ is a continuously differentiable matrix we have

$$
\begin{aligned}
0 & =\int_{t_{1}}^{t_{0}}\left(\left\langle u^{\prime}, u^{\prime}\right\rangle-\langle M u, u\rangle^{\prime}-\langle A u, u\rangle\right) d t \\
& =\int_{t_{1}}^{t_{0}}\left(\left\langle u^{\prime}, u^{\prime}\right\rangle-2\left\langle\tilde{M} u^{\prime}, u\right\rangle+\left\langle\tilde{M}^{2} u, u\right\rangle-\langle L u, u\rangle\right) d t
\end{aligned}
$$

where $\tilde{M}=\frac{1}{2}\left(M+M^{T}\right)$ and $L(t)=M^{\prime}+\tilde{M}^{2}+A$. Finally we get

$$
0=\int_{t_{1}}^{t_{0}}\left(\left\|u^{\prime}-\tilde{M} u\right\|^{2}-\langle L u, u\rangle\right) d t
$$

Since $u_{k}(t) \geqslant 0$ and $l_{i j}(t) \leqslant 0$, then both terms of the integrand in (7) are nonnegative for $t_{1} \leqslant t \leqslant t_{0}$. It follows that both of these terms must vanish for all $t \in\left[t_{1}, t_{0}\right]$. The vanishing of the first term means that $u(t)$ is a solution of $u^{\prime}=\tilde{M} u$ with $u\left(t_{1}\right)=u\left(t_{0}\right)=0$. Therefore $u(t) \equiv 0$. This is a contradiction.

REMARK. We point out that the converse of Theorem 4 will be true when $A(t)$ is symmetric, for if (1) is disconjugate there exists a nonsingular matrix solution $Y(t)$ on $[a, b]$ of $Y^{\prime \prime}+A(t) Y=0$ and wé take $M(t)=Y^{\prime} Y^{-1}$.

We raise the following question:

Question. If $A(t)$ is nonsymmetric, does the converse of Theorem 2 hold?

AcKnowledgement. The author would like to thank Professor Shair Ahmad for his valuable comments during the preparation of this paper.

\section{REFERENCES}

1. S. Ahmad and A. C. Lazar, On the components of extremal solutions of second order systems, SIAM J. Math. Anal. 8 (1977), 16-23.

2. _ An N-dimensional extension of the Sturm separation and comparison theory to a class of nonselfadjoint systems, SIAM J. Math. Anal. 9 (1978), 1137-1150. 
3. A new generalization of the Sturm comparison theorem to selfadjoint systems, Proc. Amer. Math. Soc. 68 (1978), 185-188.

4. R. L. Sternberg, Variational methods and nonoscillation theorems for systems of differential equations, Ann. Polon. Math. 21 (1969), 175-194.

5. J. C. F. Sturm, Mémoire sur les équations différentielles linéaires de second ordre, J. Math. Pures Appl. 1 (1836), 106-186.

6. A. Wintner, On the nonexistence of conjugate points, Amer. J. Math. 73 (1951), 368-380.

Department of Mathematics, Kuwait University, P. O. Box 5969, Kuwait

Current address: Faculty of Civil Engineering, University of Damascus, Damascus, Syria 